\title{
Recurrent Mixed Neuroendocrine- Non-Neuroendocrine Neoplasm in the Gallbladder: A Case Report
}

\author{
Turki Al-Fayeaa, b, c Mohammed Alzahrani a, b, d Hatim Almaghrabia, b, e \\ Abdulrahman Alghamdia, ${ }^{a}$ Kabo Mohammed ${ }^{a, b}$, \\ ${ }^{a}$ College of Medicine, King Saud Bin Abdulaziz University for Health Sciences, Jeddah, \\ Saudi Arabia; ${ }^{b}$ King Abdullah International Medical Research Center, Jeddah, Saudi Arabia; \\ 'Department of Medical Oncology, Princess Noorah Oncology Center, King Abdulaziz \\ Medical City, Jeddah, Saudi Arabia; ${ }^{d}$ Department of Surgery, King Abdulaziz Medical City, \\ Jeddah, Saudi Arabia; 'Department of Pathology and Laboratory Medicine, King Abdulaziz \\ Medical City, Jeddah, Saudi Arabia
}

\section{Keywords}

Gallbladder neoplasms · Neuroendocrine tumors · Mixed neuroendocrine-

non-neuroendocrine neoplasm

\section{Abstract}

Mixed neuroendocrine-non-neuroendocrine neoplasms (MiNEN) of the gallbladder are rare with no established therapeutic strategies. We report a case of recurrent gallbladder MiNEN from a population with a low incidence of gallbladder carcinomas, a review of the current therapeutic options, and recent updates on the nomenclature proposed by the World Health Organization in 2017.

\section{Introduction}

The gallbladder is an uncommon site for malignant neoplasms. Gallbladder carcinomas (GBCs) represent only $0.5 \%$ of all malignant tumors [1]. The global annual incidence rate is 2.2 per 100,000 population [2]. In Saudi Arabia, the incidence rate of GBCs was reported in 2014 to be lower, with 1.2 per 100,000 population in men, and 1.3 per 100,000 population in women [3]. Certain ethnicities show a higher incidence rate, for example South American Indian women (16-27/100,000) and East Indians (22/100,000) [4]. Multiple factors are considered to increase the GBC incidence rate, including gallstones, obesity, infection, female gender, Asian descent, and a low socioeconomic status [4]. 
GBCs can arise in any part of the gallbladder, with the fundus the most frequent area (60\%) [5]. Histopathologically, adenocarcinomas with their variants are the most frequent subtypes [1]. Other histological types include squamous cell carcinoma $(<3 \%)$, small-cell carcinoma, and undifferentiated carcinoma. Neuroendocrine carcinomas are a different entity representing $0.5 \%$ of all GBCs [1]. GBCs are rarely a mixture of any of the epithelial variants of neuroendocrine carcinomas. Such tumors with a neuroendocrine and non-neuroendocrine carcinoma composition are classified as mixed neuroendocrine-non-neuroendocrine neoplasms (MiNENs) [6]. The latest 2017 World Health Organization (WHO) Blue Book for the classification of gastrointestinal tumors requires that each component constitute at least $30 \%$ of the overall tumor cells [6]. This entity is extremely rare and tends to have aggressive biological behavior associated with a poor prognosis. Here, we report a new case of a gallbladder MiNEN with a brief review of the recent updates in nomenclature and the current evidence about their management.

\section{Case Presentation}

A 69-year-old Saudi woman was admitted to our hospital with a right upper quadrant pain, associated with nausea and loss of appetite for about a month. The pain was aggravated by fatty food. Her medical and surgical history included type 2 diabetes mellitus, hypertension, and dyslipidemia. There was no malignancy in the family history. The physical examination was unremarkable except for a mild tenderness over the right upper quadrant. The blood tests indicated an abnormal liver profile, including alanine aminotransferase $105 \mathrm{U} / \mathrm{L}$ (normal range 6-28), alkaline phosphatase $316 \mathrm{U} / \mathrm{L}$ (normal range 39-114), and total bilirubin $68.7 \mathrm{Umol} / \mathrm{L}$ (normal range 2.1-15.5). An abdominal ultrasonography revealed a distended gallbladder $(15 \times 5 \mathrm{~cm})$ with an impacted stone at the neck of the common bile duct with intrahepatic duct dilatation. On the magnetic resonance cholangiopancreatography an infiltrative pattern was noticed at the hepatic hilum forming a circumferential thickening of the cystic duct, neck of the gallbladder, and the proximal common bile duct. A marked elevation of carbohydrate antigen 19-9 was present (1,880 IU/mL, normal range $0-47)$. She underwent an extended open cholecystectomy with a proximal bile duct excision, porta hepatis dissection, and hepatojejunostomy.

The pathological evaluation revealed a tumor consisting of two populations of neoplastic cells. The first consisted of tumor cells arranged in tubules and solid areas with cells showing round nuclei, prominent nucleoli, and a moderately vacuolated cytoplasm. This component represented a moderately differentiated adenocarcinoma, which constituted about $60 \%$ of the tumor. The second population of cells was formed of solid sheets with extensive areas of necrosis and scattered viable areas. The viable areas consisted of sheets of smaller-sized cells with salt-and-pepper chromatin and scant cytoplasm (Fig. 1). No other histological components were identified, in spite of extensive sampling of the tumor at 1 section $/ \mathrm{cm}$. Immunohistochemical stains showed the second population expressing neuroendocrine markers (synaptophysin and chromogranin; Ventana BenchMark Ultra, Ventana Medical Systems, Tucson, AZ, USA). The first population was negative for the two immunohistochemical stains (Fig. 2). The Ki-67 score with immunohistochemistry was positive in $90 \%$ of the small-cell component, significantly higher than the Ki-67 score of 70\% in the adenocarcinoma component.

Subsequent to the pathological report of MiNEN, a full radiological and laboratory workup for possible metastasis was performed but none was revealed. Twelve weeks later, she was admitted with a recurrent local extensive disease and ascending cholangitis. An abdominal CT scan (Fig. 3) showed extensive soft tissue thickening at the level of the gallbladder bed at 


\section{Case Reports in Oncology}

\begin{tabular}{l|l}
\hline Case Rep Oncol 2021;14:411-417 \\
\hline DOI: 10.1159/000513031 & $\begin{array}{l}\text { ○ 2021 The Author(s). Published by S. Karger AG, Basel } \\
\text { www.karger.com/cro }\end{array}$ \\
\hline
\end{tabular}

Al-Fayea et al.: A Case of Recurrent Mixed Neuroendocrine-Non-Neuroendocrine Neoplasm

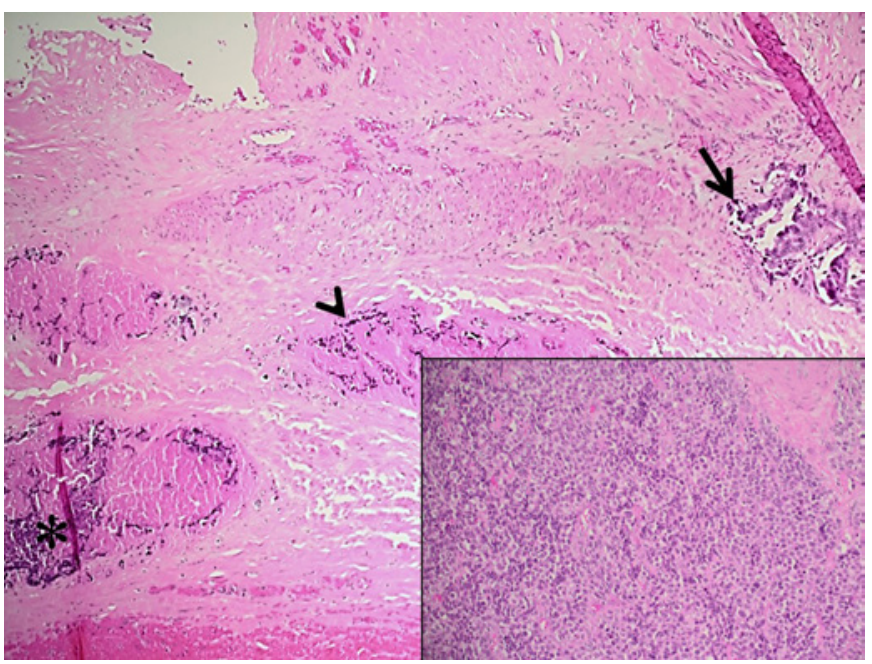

Fig. 1. Histological section at the interface between adenocarcinoma and neuroendocrine components. Tumor cells arranged in a tubular architecture, a feature of adenocarcinoma, infiltrate the muscularis propria of the gallbladder wall (black arrow). The neuroendocrine component at the interface shows infiltration of the gallbladder wall with morphologically distinct higher-grade tumor with areas of necrosis (arrow head), and few viable areas (asterisk). HE stain, $\times 100$. Inset Viable areas of neuroendocrine component consisting of a nest of tumor cells in diffuse sheets formed of small round cells with round nuclei exhibiting salt-andpepper chromatin with frequent mitosis (>20 mitosis/hpf) and apoptosis. HE stain, $\times 200$.
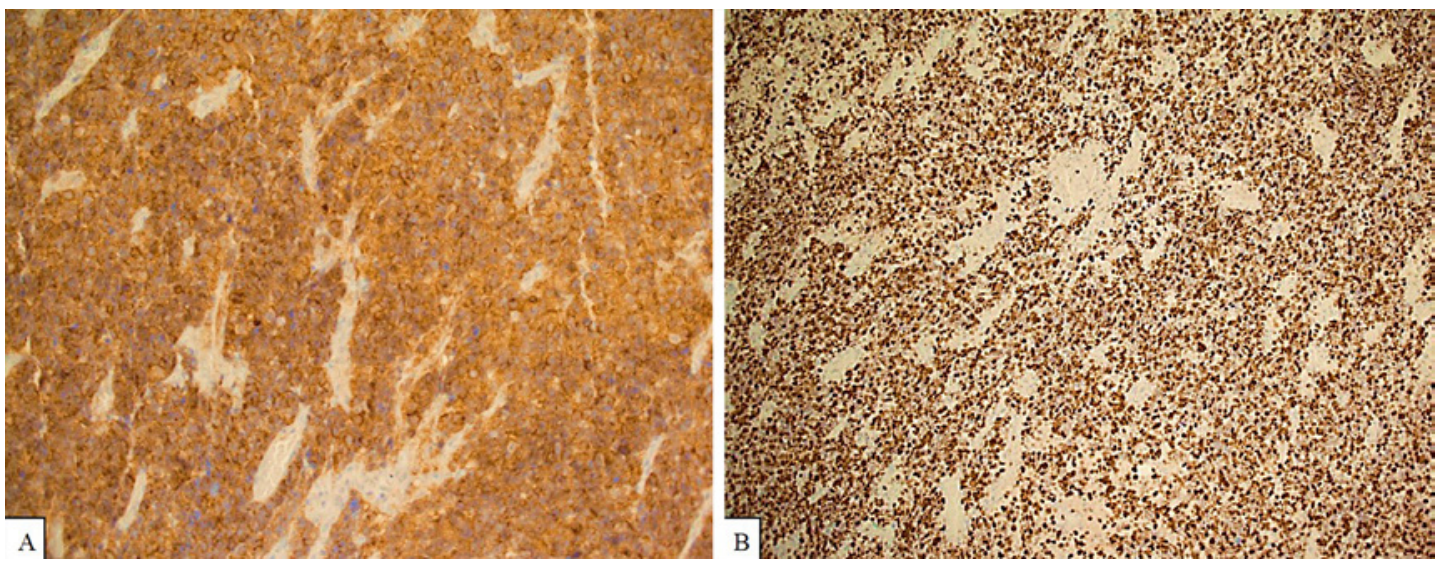

Fig. 2. Immunohistochemistry of the diffuse sheets of small-size tumor cells exhibit strong granular cytoplasmic and membranous positivity for synaptophysin (A) and nuclear positivity for Ki-67 in around $70 \%$ of tumor cells (B). Original magnification, $\times 200$.

the region of the hepatojejunostomy, which demonstrated infiltration into segment 5 of the liver, which surrounds and infiltrates the confluence of the right and left hepatic ducts, causing significant intrahepatic biliary channel dilatation. The multidisciplinary team decision was not to start systemic therapy as the patient had a poor performance status, indicating possible poor tolerance and detrimental treatment effects. The patient was referred to palliative care services. 


\section{Case Reports in Oncology}

Case Rep Oncol 2021;14:411-417

\begin{tabular}{l|l}
\hline DOI: 10.1159/000513031 & (c) 2021 The Author(s). Published by S. Karger AG, Basel
\end{tabular} www.karger.com/cro

Al-Fayea et al.: A Case of Recurrent Mixed Neuroendocrine-Non-Neuroendocrine Neoplasm
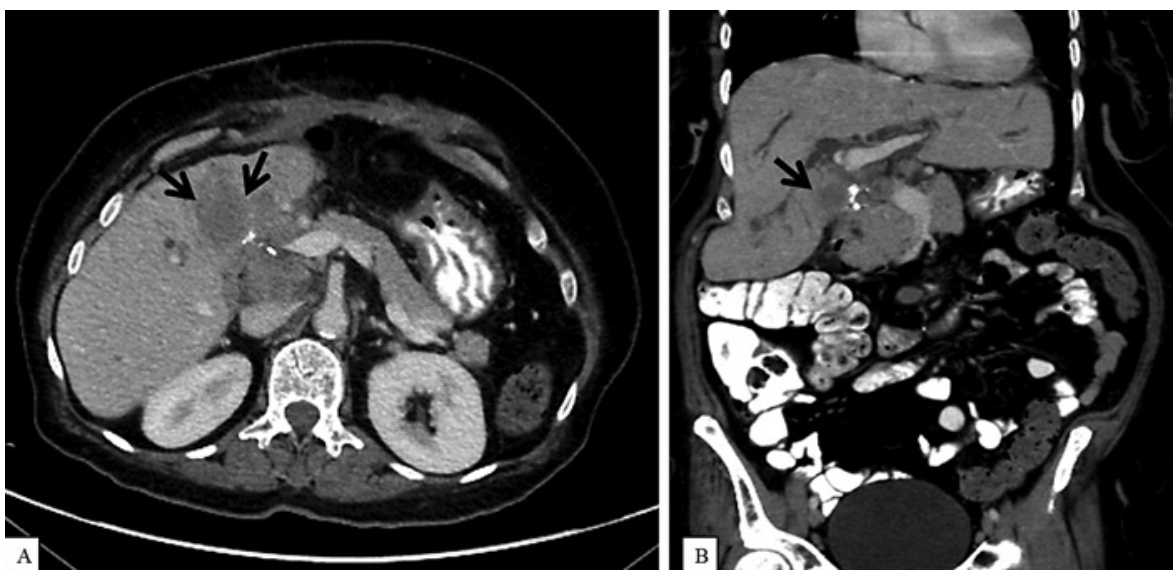

Fig. 3. A contrast-enhanced CT scan of the abdomen (axial, A, and coronal, B) post-gallbladder resection and common bile duct stent insertion, showing an infiltrative soft tissue mass (black arrow) at the gallbladder bed and porta hepatis around the stent and portal vein.

\section{Discussion}

The concept of dual differentiation (exocrine and neuroendocrine) in gastrointestinal tumors was first introduced in 1924 [7]. In 1987, Lewin suggested a classification of mixed differentiation tumors including 3 classes: combined, collision, and amphicrine [8]. In 2010, the WHO classification for tumors considered any tumor with exocrine and nonendocrine components as mixed adenoneuroendocrine carcinoma (MANEC) [9]. However, in 2017, this nomenclature was changed from MANEC to MiNENs [6].

The redefined term avoids the confusion resulting from the MANEC terminology. The MANEC terminology only considered the adenocarcinoma component and excluded other non-adenocarcinoma components such as acinar or squamous cell carcinoma. In addition, MANEC confuses the mixed adenocarcinoma and neuroendocrine component with other entities such as goblet cell carcinoid (adenocarcinoid), which are less aggressive tumors. The term MiNENs is more inclusive and encompasses other non-adenocarcinoma components that can be present concurrently with neuroendocrine tumor components.

The MiNENs terminology requires that each individual component contain at least 30\% of the total tumor cell population [6]. Our case histopathology consisted of an adenocarcinoma component occupying $60 \%$ of the tumor, with the remaining $40 \%$ occupied by the small cell neuroendocrine component. No other squamous cell or acinar components were identified. This cut-off value has been assigned arbitrarily to indicate the tumor load that is thought to affect the prognosis. When the neuroendocrine component does not comply with this condition (i.e., $<30 \%$ ), the tumor is known as a non-neuroendocrine neoplasm with a focal neuroendocrine component.

Generally, neuroendocrine tumors are assumed to stem from neural crest cells, frequently present in the respiratory and gastrointestinal tracts [1]. However, the origin of the neuroendocrine component in the gallbladder is uncertain because neuroendocrine cells are infrequently present in the gallbladder wall. Many theories have been proposed to describe the histogenesis of these tumors in the gallbladder. One theory suggests the independent development of each component in the metaplasia-dysplasia-carcinoma sequence from dysplastic gastrointestinal or biliary epithelium [10]. A second theory is that MiNENs are the result of the trans-differentiation of adenocarcinomas $[10,11]$ and, lastly, the possibility that they originate from cancerous stem cells [1]. 
Patients with gallbladder MiNEN usually present with non-specific clinical manifestations such as abdominal pain and jaundice. Laboratory and imaging tests, including ultrasound, abdominal CT scan, and magnetic resonance imaging, can be suggestive of ongoing neoplastic changes. In a case series of neuroendocrine tumors in the gallbladder, CT findings indicative of neuroendocrine tumors indicated the existence of large heterogeneous masses inside the gallbladder with enlarged metastatic lymph nodes [12].

MiNENs cannot be confirmed preoperatively as a pathological examination with immunohistochemical staining for markers, most importantly synaptophysin and chromogranin, is required. Synaptophysin is a glycoprotein component of neuroendocrine secretory granules. A broad-spectrum neuroendocrine marker, SY38 antibody, is an immunohistochemical marker of synaptophysin, which has a higher sensitivity for neuroendocrine differentiation but a lower specificity than chromogranin. Positivity for synaptophysin alone is not enough to determine the neuroendocrine lineage. Within the proper histomorphological background, it is a useful marker of neuroendocrine differentiation. In one study, small-cell lung carcinomas were stained with synaptophysin in $79 \%$ of the cases, whereas chromogranin was positive in $47-60 \%$ of the cases $[13,14]$.

Our patient was diagnosed with recurrent extensive local disease. The non-specific symptoms, with the aggressive behavior of such tumors, delay the diagnosis in the early stages and worsen the prognosis. Unfortunately, due to the shortage of literature about locally recurrent gallbladder MiNEN, appropriate treatment is still debatable and usually personalized depending on each case's clinical circumstances. Hepatectomy is a potential cure. Currently, there are two main management pathways. The first is the latest WHO recommendation to treat MiNEN as adenocarcinomas or, secondly, to treat such tumors on the basis of which histological component is more aggressive, which is more frequently followed in biliary MiNENs due to the grade heterogeneity of both components $[9,15]$.

A patient with localized, histologically proven MiNENs with a high Ki-67, indicating aggressive disease, should consider surgery followed by adjuvant chemotherapy. Data of using systemic chemotherapy in recurrent/advanced MiNENs are lacking. The chemotherapy regimens used are usually extrapolated from larger series treating gastroenteropancreatic poorly differentiated neuroendocrine carcinomas [16-18]. Patients with advanced poorly differentiated gastroenteropancreatic neuroendocrine carcinomas are often treated as small cell carcinoma and have a high response to chemotherapy. Such a patient should be offered systemic chemotherapy as soon as they are clinically fit, since the performance status is considered an important prognostic factor. Other predictive factors for the response to chemotherapy include a higher level of Ki-67 [17]. Platinum (either cisplatin or carboplatin) and etoposide is a frequently used regimen with a median survival of 11 months $[17,18]$. Other regimens include irinotecan and platinum, proven effective in patients of Asian descent [19]. Similar to small-cell lung cancer, patients with advanced MiNENs with a poorly differentiated neuroendocrine component tend to relapse quickly and the response to subsequent therapy is poor. Options include topotecan as a single agent versus a rechallenge with platinum and etoposide, if the relapse is more than 3 months after the first-line therapy [20]. Whenever it is possible, referral to a specialized center with a high volume of the specific disease may be a good alternative to improve the outcome.

\section{Conclusion}

We report a rare case of recurrent gallbladder MiNEN from a low-incidence population of GBCs, using the new 2017 WHO classification. More documentation of this rare entity of tumors would broaden our knowledge of its morphological types and direct future studies to investigate the histogenesis of these tumors, optimizing the current treatment.

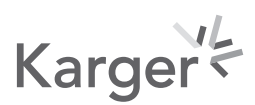




\section{Case Reports in Oncology}

Case Rep Oncol 2021;14:411-417

\begin{tabular}{l|l}
\hline DOI: $10.1159 / 000513031$ & ○ 2021 The Author(s). Published by S. Karger AG, Basel
\end{tabular} www.karger.com/cro

Al-Fayea et al.: A Case of Recurrent Mixed Neuroendocrine-Non-Neuroendocrine Neoplasm

\section{Statement of Ethics}

Written informed consent was obtained from the patient to publish this case and any accompanying images, as well as approval from the Institutional Review Board.

\section{Conflict of Interest Statement}

The authors declare no conflicts of interest.

\section{Funding Sources}

The authors produced this manuscript without any funding.

\section{Author Contributions}

T.A.-F., M.A., K.M.., and A.A. collected, analyzed, and interpreted the data. H.A. performed the histological analysis. T.A.-F., M.A., H.A., and A.A. drafted the manuscript. All authors critically revised the manuscript and approved the final version.

\section{References}

1 Mondolfi AEP, Slova D, Fan W, Attiyeh FF, Afthinos J, Reidy J, et al. Mixed adenoneuroendocrine carcinoma (MANEC) of the gallbladder: a possible stem cell tumor? Pathol Int. 2011;61(10):608-14.

2 Olivares LV. Cancer of the gallbladder - Chilean statistics. Ecancermedicalscience. 2016;10:704-8.

3 Al-Eid H, Quindo MA. Cancer incidence report Saudi Arabia 2010 [Internet]. 2014. Available from: http:// www.chs.gov.sa/Ar/mediacenter/NewsLetter/2010 Report (1).pdf.

4 Hundal R, Shaffer EA. Gallbladder cancer: epidemiology and outcome. Clin Epidemiol. 2014;6:99-109.

5 Shaffer EA. Gallbladder cancer: the basics. Gastroenterol Hepatol. 2008;4(10):737-41.

6 Inzani F, Petrone G, Rindi G. The New World Health Organization Classification for Pancreatic Neuroendocrine Neoplasia. Endocrinol Metab Clin North Am. 2018;47(3):463-70.

7 Cordier R. Les cellules argentaffines dans les tumeurs intestinales. Arch Intern Med. 1924;1:5.

8 Lewin K. Carcinoid Tumors and the Mixed (Composite) Glandular-Endocrine Cell Carcinomas. Am J Surg Pathol. 1987;11 Suppl 1:71-86.

9 Bosman F, Carneiro F, Hruban R, Theise N. WHO classification of tumours of the digestive system. 4th ed. Lyon: International Agency for Research on Cancer; 2010.

10 Alemana C, Alemana C. Preneoplastic lesions in gallbladder cancer. J Surg Oncol. 2006; 93:615-23.

11 Nakajo S, Yamamoto M, Tahara E. Morphometrical analysis of gall-bladder adenoma and adenocarcinoma with reference to histogenesis and adenoma-carcinoma sequence. Virchows Arch A Pathol Anat Histopathol. 1990; 417(1):49-56.

12 El Fattach H, Guerrache Y, Eveno C, Pocard M, Kaci R, Shaar-Chneker C, et al. Primary neuroendocrine tumors of the gallbladder: ultrasonographic and MDCT features with pathologic correlation. Diagn Interv Imaging. 2015;96(5):499-502.

13 Gosney JR, Gosney MA, Lye M, Butt SA. Reliability of commercially available immunocytochemical markers for identification of neuroendocrine differentiation in bronchoscopic biopsies of bronchial carcinoma. Thorax. 1995;50(2):116-20.

14 Kayser K, Schmid W, Ebert W, Wiedenmann B. Expression of neuroendocrine markers (neuronspecific enolase, synaptophysin and bombesin) in carcinoma of the lung. Pathol Res Pract. 1988;183(4):412-7.

15 Rosa S La, Marando A, Sessa F, Capella C. Mixed adenoneuroendocrine carcinomas (MANECs) of the gastrointestinal tract: an update. Cancers. 2012;4(1):11-30.

16 Strosberg JR, Coppola D, Klimstra DS, Phan AT, Kulke MH, Wiseman GA, et al. The NANETS Consensus Guidelines for the Diagnosis and Management of Poorly Differentiated (High-Grade) Extrapulmonary Neuroendocrine Carcinomas. Pancreas. 2010;39(6):799-800. 
17 Sorbye H, Welin S, Langer SW, Vestermark LW, Holt N, Osterlund P, et al. Predictive and prognostic factors for treatment and survival in 305 patients with advanced gastrointestinal neuroendocrine carcinoma (WHO G3): the NORDIC NEC study. Ann Oncol. 2013;24(1):152-60.

18 Iwasa S, Morizane C, Okusaka T, Ueno H, Ikeda M, Kondo S, et al. Cisplatin and etoposide as first-line chemotherapy for poorly differentiated neuroendocrine carcinoma of the hepatobiliary tract and pancreas. Jpn J Clin Oncol. 2010 Apr;40(4):313-8.

19 Yamaguchi T, Machida N, Morizane C, Kasuga A, Takahashi H, Sudo K, et al. Multicenter retrospective analysis of systemic chemotherapy for advanced neuroendocrine carcinoma of the digestive system. Cancer Sci. 2014; 105(9):1176-81.

20 O'Brien ME, Ciuleanu TE, Tsekov H, Shparyk Y, Cuceviá B, Juhasz G, et al. Phase III trial comparing supportive care alone with supportive care with oral topotecan in patients with relapsed small-cell lung cancer. J Clin Oncol. 2006;24(34):5441-7. 